\title{
Using the Local Phase of the Magnitude of the Local Structure Tensor for Image Registration
}

\author{
Anders Eklund $^{1,2}$, Daniel Forsberg ${ }^{1,2,3}$, \\ Mats Andersson ${ }^{1,2}$, and Hans Knutsson ${ }^{1,2}$ \\ 1 Division of Medical Informatics, Department of Biomedical Engineering \\ ${ }^{2}$ Center for Medical Image Science and Visualization (CMIV) \\ Linköping University, Linköping, Sweden \\ 3 Sectra Imtec, Linköping, Sweden
}

\begin{abstract}
The need of image registration is increasing, especially in the medical image domain. The simplest kind of image registration is to match two images that have similar intensity. More advanced cases include the problem of registering images of different intensity, for which phase based algorithms have proven to be superior. In some cases the phase based registration will fail as well, for instance when the images to be registered do not only differ in intensity but also in local phase. This is the case if a dark circle in the reference image is a bright circle in the source image. While rigid registration algorithms can use other parts of the image to calculate the global transformation, this problem is harder to solve for non-rigid registration. The solution that we propose in this work is to use the local phase of the magnitude of the local structure tensor, instead of the local phase of the image intensity. By doing this, we achieve invariance both to the image intensity and to the local phase and thereby only use the structural information, i.e. the shapes of the objects, for registration.
\end{abstract}

\section{Introduction}

Image registration is needed in a lot of applications. One example is medical imaging where image registration is necessary to for example be able to compare images of the brain before and after surgery. A problem with the image modalities used in medical imaging is that the images produced often differ significantly in intensity. The most common approach to register images of different intensity is to maximize the mutual information (MI) between the images 1110. While this approach is sufficient to handle many registration problems, it is not very hard to create a set of test images where the mutual information approach fails, such an example is given in Fig. 1.

The main problem with the mutual information approach, and many other similarity measures, is that they are based on the intensity of the image. A better approach is to use the local phase $88|9| 1 / 4] 2] 13$. In this work we take the phase idea one step further and use the local phase of the magnitude of the local structure tensor, instead of the local phase of the image intensity. Local phase is commonly estimated by using quadrature filters 3 . 

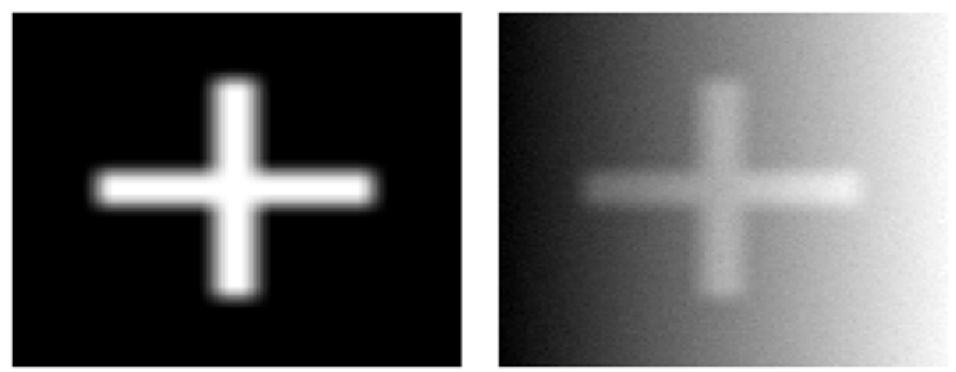

Fig. 1. Two simple test images for which intensity based registration algorithms fail

\section{Methods}

\subsection{Quadrature Filters and Local Phase}

A quadrature filter is a complex valued filter for combined edge and line detection. The real part of the filter, which is even, detects lines and the imaginary part, which is odd, detects edges. The magnitude of the complex filter response is an estimate of the phase invariant signal intensity and the phase determines whether there is an edge or a line and what kind of line or edge. We use lognormal quadrature filters $Q$, which in the Fourier domain are expressed as two polar separable functions $R$, and $D$.

$$
\begin{gathered}
Q_{k}(u)=R(\|u\|) D_{k}(u) \\
R(\|u\|)=e^{C \ln ^{2}\left(\frac{\|(u)\|}{u_{0}}\right)} \quad C=\frac{-4}{B^{2} \ln (2)}
\end{gathered}
$$

Since the phase concept is only valid if we define a direction of the signal, we construct quadrature filters with different directions. The directions are defined such that

$$
D_{k}(u)=\left\{\begin{array}{cc}
\left(u^{T} \hat{n}_{k}\right)^{2} & u^{T} \hat{n}_{k}>0 \\
0 & \text { otherwise }
\end{array}\right.
$$

We use four quadrature filters with the directions, $\Psi_{k} \in\left\{0^{0}, 45^{0}, 90^{0}, 135^{0}\right\}$. The complex filter response $q$ is an estimate of a bandpass filtered version of the analytical signal

$$
q=A \cdot(\cos (\phi)+i \cdot \sin (\phi))=A \cdot e^{i \phi}
$$

with magnitude $A$ and phase $\phi$. For image registration applications, it is important that the filters have a nice and smooth phase. This can be obtained by optmizing the filters in the spatial domain and the frequency domain at the same time 7]. 

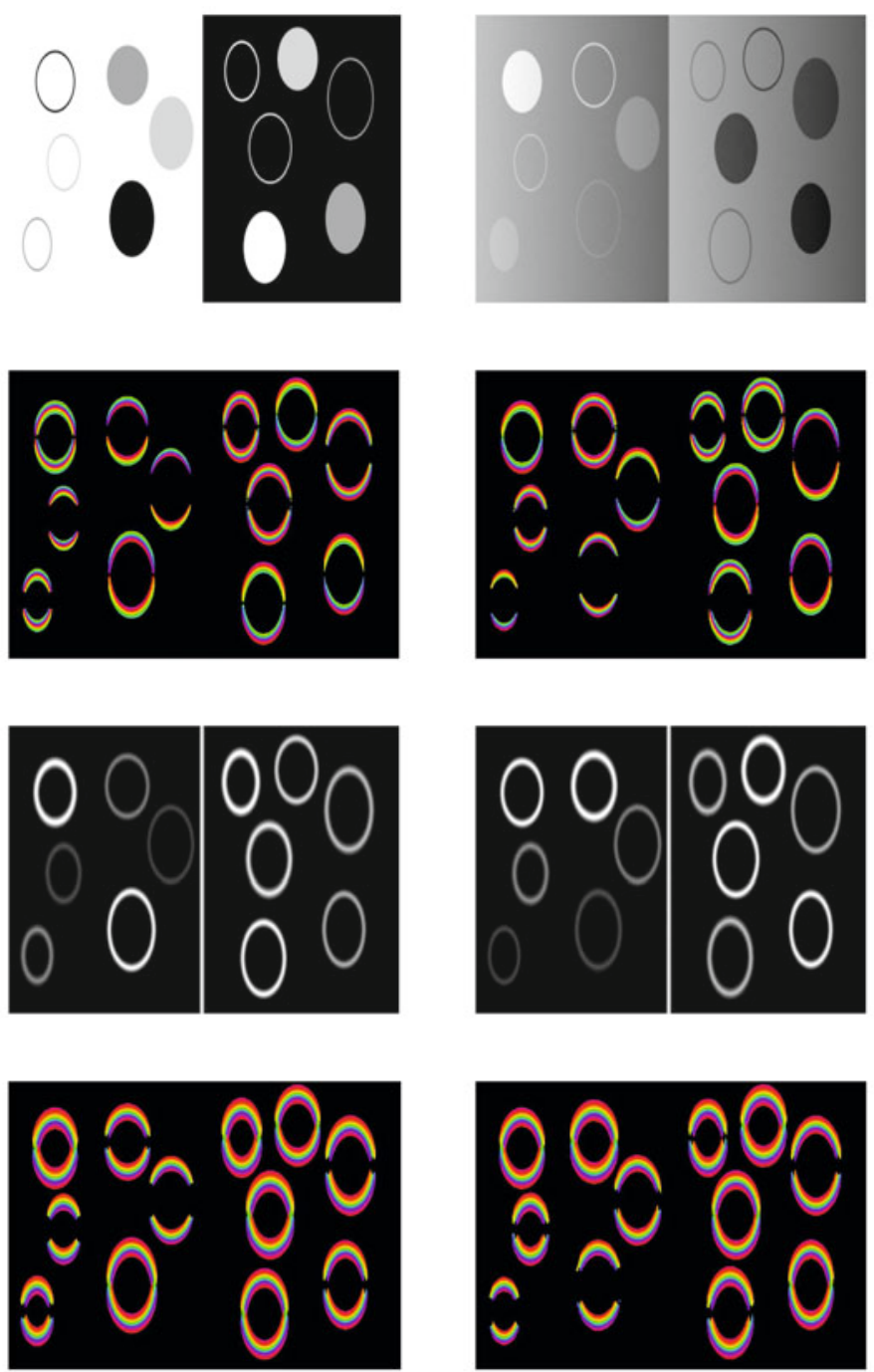

Fig. 2. First row left: A test image with all the kinds of phase for three different intensities. First row right: The test image with modified intensity and phase, a shading from right to left and noise. Second row left: The phase, in the y-direction, of the original test image. Second row right: The phase of the modified test image. The phase is only invariant to a change in intensity. Third row left: The tensor magnitude of the original test image. Third row right: The tensor magnitude of the modified test image. The tensor magnitude is only invariant to a change in phase. Fourth row left: The phase of the tensor magnitude, in the y-direction, of the original test image. Fourth row right: The phase of the tensor magnitude of the modified test image. The phase of the tensor magnitude is invariant both to change in intensity and to change in phase and thereby we can register the images by using the structural information only. Since quadrature filters are bandpass filters, they are robust to low frequencies, e.g. shadings, and high frequencies, e.g. noise. 


\subsection{The Local Structure Tensor}

The local structure tensor was introduced by Knutsson 5 to represent the local structure in images and volumes. For an image it is a $2 \times 2$ matrix in each pixel. The structure tensor can for example be used for adaptive filtering, to steer the orientation of enhancement filters. The tensor can be constructed by using the complex valued filter responses $q$ from quadrature filters. We sum over all filters $k$ and multiply the magnitude of the filter response with the outer product $N_{k}$ of the filter direction vector $\hat{n}_{k}, I$ is the identity matrix.

$$
T=\sum_{k}\left|q_{k}\right|\left(\frac{4}{3} N_{k}-\frac{1}{3} I\right), \quad T=\left(\begin{array}{ll}
t_{1} & t_{2} \\
t_{2} & t_{3}
\end{array}\right)
$$

The magnitude of the tensor is given by

$$
|T|=\sqrt{t_{1}^{2}+2 t_{2}^{2}+t_{3}^{2}}
$$

\subsection{Local Phase of the Magnitude of the Local Structure Tensor}

The problem with using the local phase for image registration is that we might have structures in the images that are similar but have different phase, for example a white circle on a black background in one image and a black circle on a white background in another image. The mutual information of the phase can handle this, as long as the mappings are consistent, but not if we want to map a dark line to a bright line in one part of the image and to map a dark line to a dark edge in another part of the image. To fully take advantage of the structural information, a better approach is to use a signal representation that is invariant both to a change in intensity and to a change in the local phase. The magnitude of the local structure tensor is invariant to a change in phase, since the orientation for a line and an edge is the same, but not to a change in intensity. The local phase on the other hand is only invariant to a change in intensity. By using the local phase of the local structure tensor magnitude, we achieve a representation that is invariant both to a change in intensity and to a change in phase. An example of this is given in Fig. 2.

\section{Results}

We have made a comparison between mutual information of the intensity, mutual information of the phase of the intensity and mutual information of the phase of the tensor magnitude. One image was rotated between -30 degrees and 30 degrees while the other remained still. We divided the 60 degree interval into 101 evaluations. As test images, we used the synthetic test images shown in Fig. 2 and four MRI images, shown in Fig 3. These MRI images differ significantly 


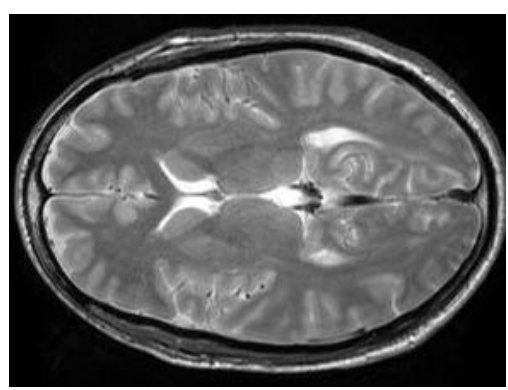

Slice 1

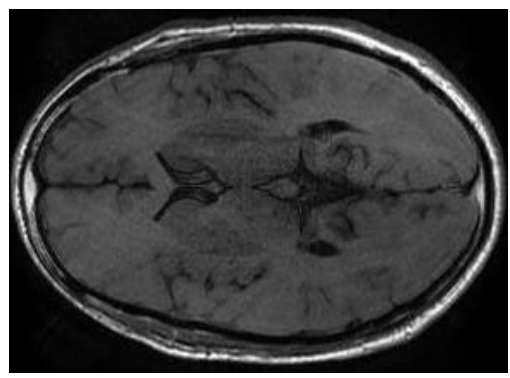

Slice 3

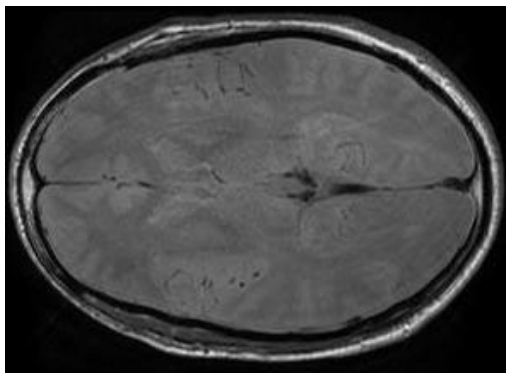

Slice 2

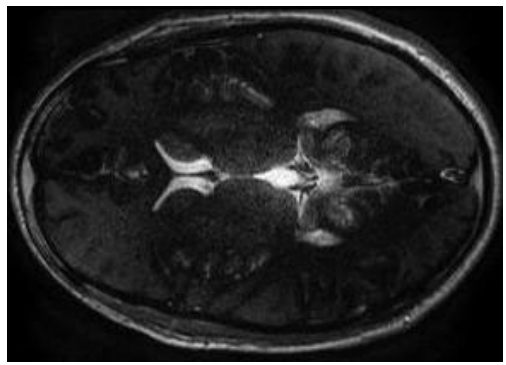

Slice 4

Fig. 3. Four MRI slices that are collected from the same location of one subject. The slices were acquired with a $1.5 \mathrm{~T}$ MR scanner. Different scanner settings were used to generate the different slices. The purpose of the image acquisition was pixel-wise quantification of physical parameters, based on signal intensity changes as a function of MR scanner settings. MRI quantification requires a motionless subject and image registration improves the result (courtesy of J.B.M. Warntjes, CMIV, Linköping, Sweden).

in intensity and for some parts of the image, but not all, the phase has been inverted. We normalized all similarity measures to have a maximum of 1 , to easier compare the slopes. The resulting plots of the similarity measures as function of rotation are given in Fig. 4.

To show that the local phase of the tensor magnitude is better than the local phase of the image intensity for image registration, we performed non-rigid registration on the two synthetic test images given in Fig. 2 and on two of the MRI images given in Fig. 3. The MRI images are used for quantitative MRI, in order to measure physical properties 12 . To obtain good measurements it is important that the images are registered. The registration algorithm used is the Morphon [6], which is a phase based non-rigid registration algorithm.

The results of the registration of the synthetic test images are shown in Fig. 5. The results of the registration of the MRI images are shown in Fig. 6. 

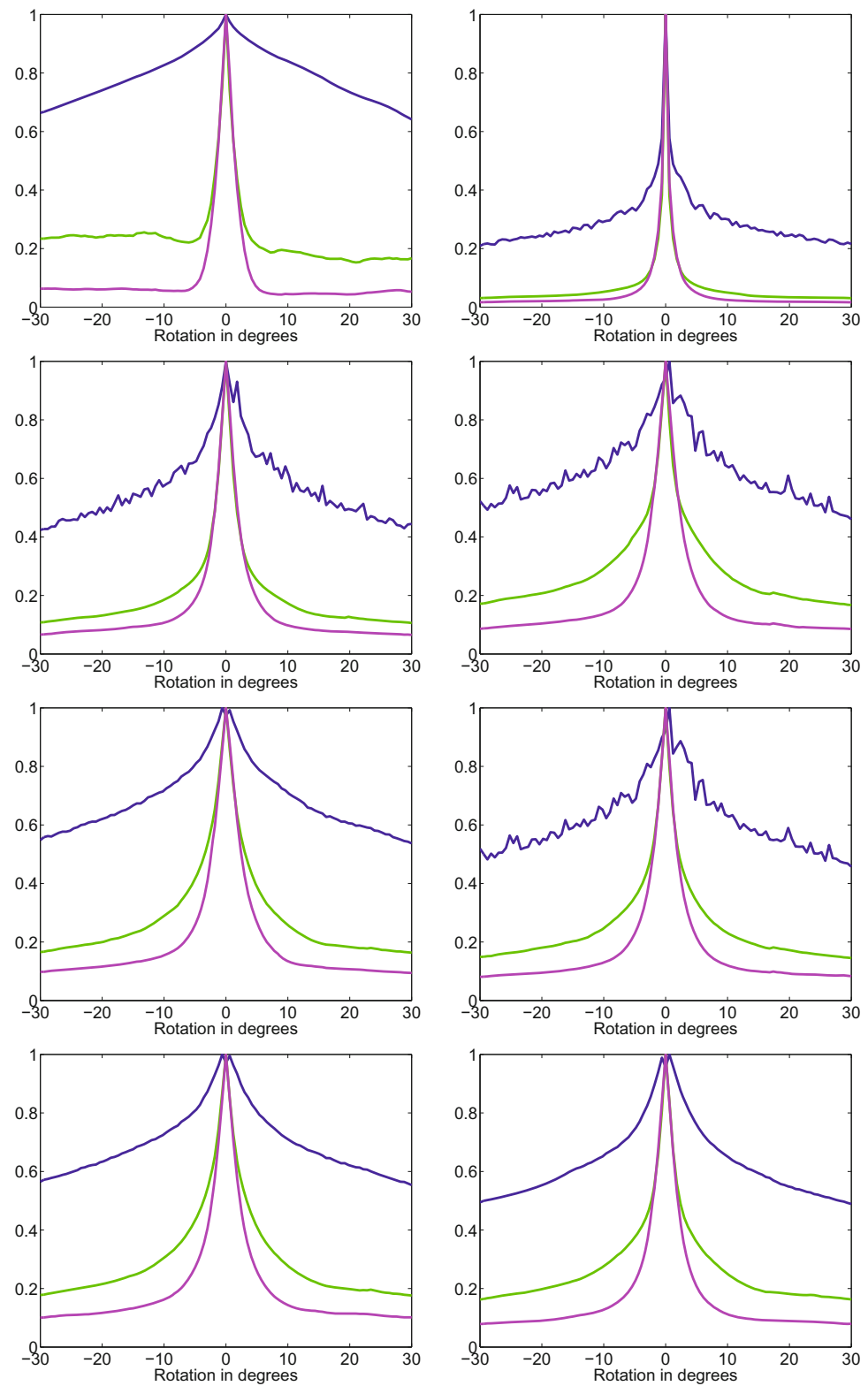

Fig. 4. Similarity measures as function of rotation between two images. The blue line is the mutual information of the intensity values, the green line is the mutual information of the phase of the intensity and the pink line is the mutual information of the phase of the tensor magnitude. First row left: Original test image and modified test image, from Fig. 2. First row right: Slice 1 and slice 1. Second row left: Slice 1 and slice 2. Second row right: Slice 1 and slice 3. Third row left: Slice 1 and slice 4 . Third row right: Slice 2 and slice 3. Fourth row left: Slice 2 and slice 4. Fourth row right: Slice 3 and slice 4 . 

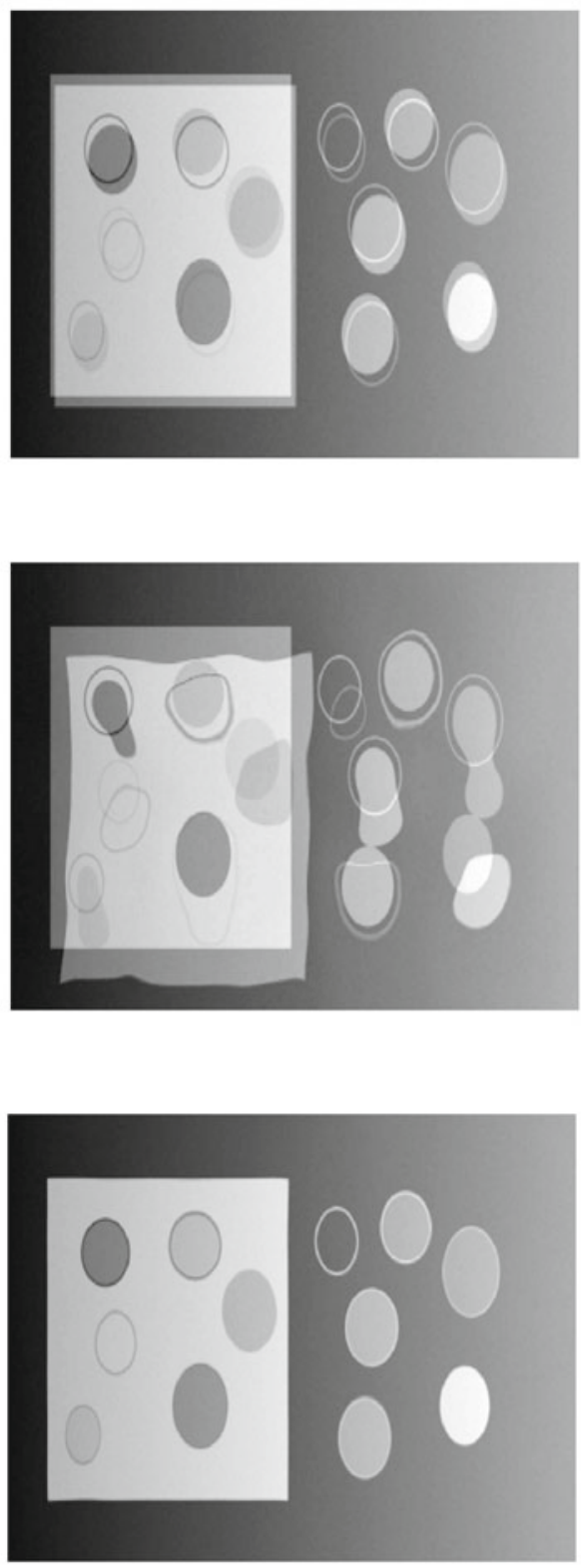

Fig. 5. Top: The original intensity difference between the original test image and the altered test image. The images have been shifted 3 pixels in the $\mathrm{x}$-direction and 5 pixels in the y-direction. Middle: The intensity difference after the non-rigid registration with the phase of the intensity. The altered phases confuse the registration algorithm and the registration does not work at all. Bottom: The intensity difference after the nonrigid registration with the phase of the tensor magnitude. By using the phase of the tensor magnitude we achieve invariance both to the intensity and to the local phase and now the registration works correctly. 

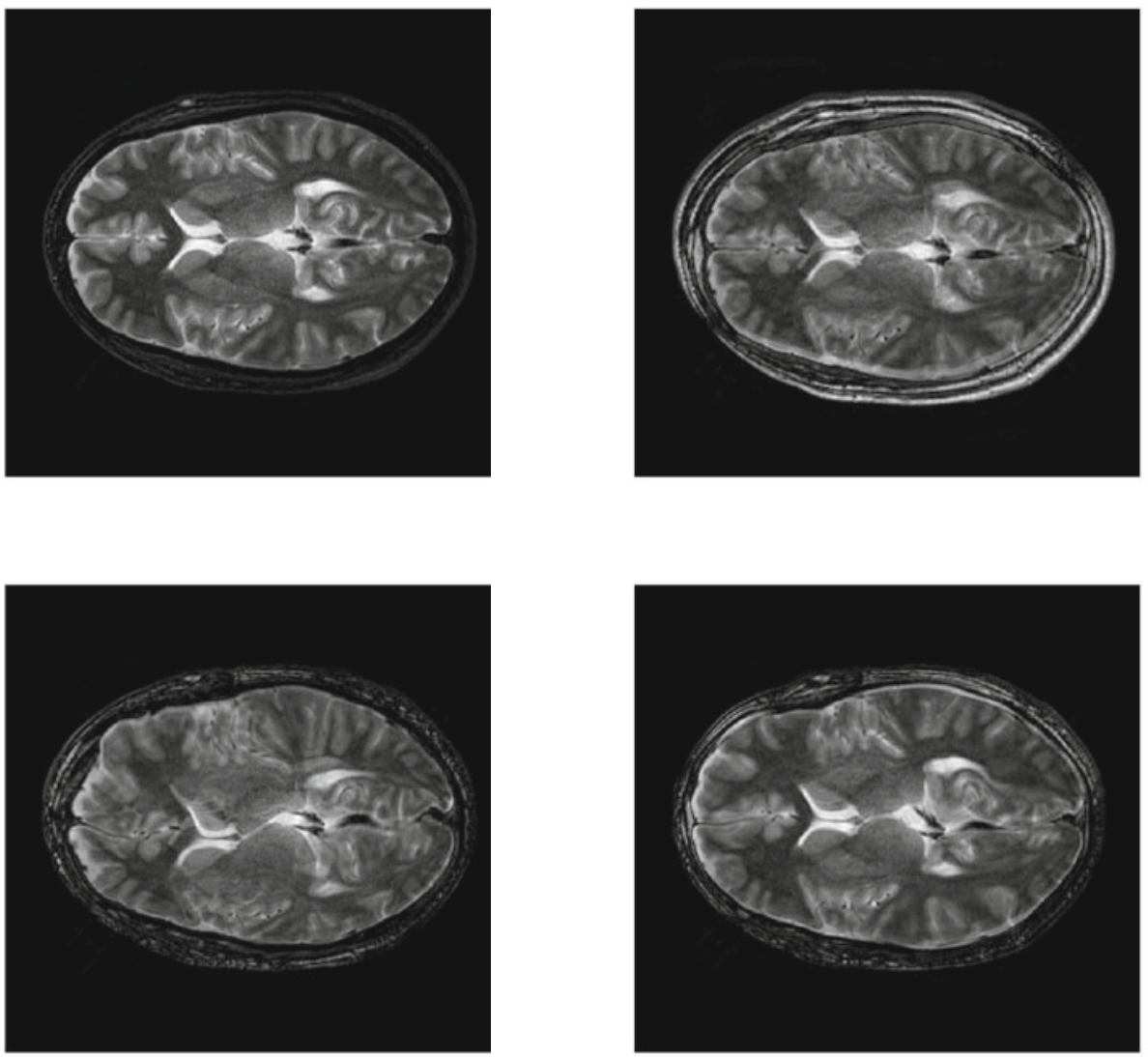

Fig. 6. Top left: The original absolute intensity difference between slice 3 and slice 1 . Top right: The absolute intensity difference between slice 3 and slice 1 after shifting the images 6 pixels in the $\mathrm{x}$-direction and 7 pixels in the $\mathrm{y}$-direction. Bottom left: The absolute intensity difference after the non-rigid registration with the phase of the intensity. The registration works rather well for the fat border surrounding the brain but not at all for the ventricles, where the phase is inverted. Bottom right: The absolute intensity difference after the non-rigid registration with the phase of the tensor magnitude. Now the registration works for the ventricles as well.

\section{Discussion}

The similarity measure comparison in Fig. 4 clearly shows that our new similarity measure decays faster than the other similarity measures. The biggest difference is however between the intensity and the local phase.

As can be seen in Fig. 5, the local phase of the intensity does not work at all to register the synthetic test images. By instead using the local phase of the tensor magnitude we achieve invariance to the local phase and can use the structural information, i.e. the shape of the objects, for registration. 
For the MRI images similar results are obtained, the local phase of the intensity works rather well for the fat border surrounding the brain, but it does not work at all for the ventricles since they are bright in one image and dark in the other. With the local phase of the tensor magnitude the registration works for the ventricles as well. For rigid registration, the local phase of the intensity works as well as the local phase of the tensor magnitude. The reason for this is that the fat border surrounding the brain is the same in all the images. If the fat border is removed, the rigid registration with the local phase of the intensity fails, while the registration works if the local phase of the tensor magnitude is used.

A major advantage with the presented approach is that existing image registration algorithms can be used without modification. Instead of using the reference image and the source image as inputs, the tensor magnitude of the reference image and the tensor magnitude of the source image are used. The found displacement field is then applied to the source image. The increase in processing time is thus rather small.

\section{Acknowledgement}

This work was supported by the Linnaeus center CADICS, funded by the Swedish research council.

We thank Marcel Warntjes et al. at CMIV for letting us use their MRI images to test our registration algorithm with.

\section{References}

1. Dalvi, R., Abugharbieh, R., Pickering, M., Scarvell, J., Smith, P.: Registration of $2 \mathrm{D}$ to $3 \mathrm{D}$ joint images using phase-based mutual information. In: Proceedings of SPIE (2007)

2. Eklund, A., Andersson, M., Knutsson, H.: Phase based volume registration using CUDA. In: IEEE International Conference on Acoustics, Speech and Signal Processing (ICASSP), pp. 658-661 (2010)

3. Granlund, G., Knutsson, H.: Signal Processing for Computer Vision. Kluwer Academic Publishers, Dordrecht (1995) ISBN 0-7923-9530-1

4. Hemmendorff, M., Andersson, M., Kronander, T., Knutsson, H.: Phase-based multidimensional volume registration. IEEE Transactions on Medical Imaging 21, 1536-1543 (2002)

5. Knutsson, H.: Representing local structure using tensors. In: Scandinavian Conference on Image Analysis (SCIA), pp. 244-251 (1989)

6. Knutsson, H., Andersson, M.: Morphons: Paint on priors and elastic canvas for segmentation and registration. In: Scandinavian Conference on Image Analysis (SCIA), Joensuu (2005)

7. Knutsson, H., Andersson, M., Wiklund, J.: Advanced filter design. In: Scandinavian Conference on Image Analysis, SCIA (1999)

8. Mellor, M., Brady, M.: Non-rigid multimodal image registration using local phase. In: Barillot, C., Haynor, D.R., Hellier, P. (eds.) MICCAI 2004. LNCS, vol. 3216, pp. 789-796. Springer, Heidelberg (2004) 
9. Mellor, M., Brady, M.: Phase mutual information as similarity measure for registration. Medical Image Analysis 9, 330-343 (2005)

10. Pluim, J.P.W., Maintz, J.A., Viergever, M.A.: Mutual information based registration of medical images: a survey. IEEE Transactions on Medical Imaging 22, 986-1004 (2003)

11. Viola, P., Wells, W.: Alignment by maximization of mutual information. International Journal of Computer Vision 24, 137-154 (1997)

12. Warntjes, J., Dahlqvist, L., West, J., Lundberg, P.: Rapid magnetic resonance quantification on the brain: Optimization for clinical usage. Magnetic Resonance in Medicine 60, 320-329 (2008)

13. Wong, A., Fieguth, P.: Fast phase-based registration of multimodal data. Signal Processing 89, 724-737 (2008) 